\title{
APPENDIX
}

TO THE

\section{FOREGOING PAPER.}

\author{
Bý ALEX. C. HUTCHISON, M.D. \\ SURGEON TO THE ROYAL NAVAL HOSPITAL AT DEAL.
}

\section{SINCE I had the honour of submitting to the} Society a paper on certain diseases of the brain, I have met with an instance of a peculiarity of structure in that organ; which has not, as far as I have been able to discover, been hitherto described *; and, as it bears a striking analogy to the subject of that paper, I beg leave to subjoin the following account of it, accompanied with a brief history of the previous circumstances, as detailed by Mr. William. son, surgeon to his Majesty's ship Christian the 7th.

“ Thomas Dawson, seaman, ætatis 28, of low stature, and of a full and healthy habit; while as. sisting to get up the top-gallant masts, on the 11 th

* In the 3d vol. of the Memoirs of the Medical Society of London, page 54, Dr. Lettsom has given a long and very respectable list of auttiors whom he had consulted on affections of the bead arising from various causes. 
of March, 1813, the leading block gave way, and, his feet being entangled in the rope, he was tripped up, and his forehead struck with considerable violence on the deck. The part was contused and slightly discoloured, but the skin was scarcely abraded; he was a little stunned by the blow, but spoke sensibly soon after.

On examination, no fracture nor fissure could be discovered; his pulse was regular, and his eyes natural; he had no nausea, but bled freely from the nose, and corplained of a severe pain over the fore part of the head. He was prescribed a cathartic, and compresses wetted with Saturnine lotion were kept constantly applied to the contused part.

In the afternoon, he had slight nausea with vomiting; the headach was the same; and as the cathartic had not operated, it was directed to be repeated.

Second day.-The symptoms continued nearly the same; the bowels not being sufficiently opened, the purgative was repeated. Towards evening, febrile symptoms began to shew themselves; the pulse was increased in fulness and frequency; the skin was hot, face flushed, and eyes irritable. He had some stupor, with a disposition to sleep; the bowels were open. He was bled to twenty ounces, which entirely removed the febrile heat, and, in some degree, relieved the headach. Ever since 
the accident he had been unable to walk straight; and seemed to totter, not unlike a man intoxicated, the few times he tried that exercise.

Third day.-He said the pain was chiefly confined to the posterior part of the head; the bowels were open; there was no febrile affection, and his eyes were less irritable. A blister was applied to the nape of the neck, the pediluvium used, and he took camphorated saline draughts every four hours.

Fourth day.-There was no change, except that he rested rather better, throughout the last night, than he had done since the accident; - the camphorated saline draughts were directed to be continued.

Fifth day.-He had considerable febrile heat, with much irritability and restlessness, and some thirst; his face was flushed, pulse frequent and rather hard; respiration hurried and tongue white. He passed his urine unconsciously last night and this morning; pain now, he says, chiefly confined to the fore part of the head. He was bled again to 24, ounces, and, during this operation, the pulse kept its fulness. Immediately after the bleeding, he said his head was much relieved; the febrile heat, flushed face, and hurried respiration were certainly diminished, and he seemed more tranquil. The blood exhibited a dense buffy coat. Within a very few hours afterwards every symptom 
increased,-his pulse became small and frequent, -his pupils dilated,-low muttering delirium and restlessness succeeded, and at half past twelve o'clock, on the sixth day, (the 16th of March) he expired without the least convulsive struggle.

It appears, by the concurring reports of his messmates, that he was always a healthy man, of a lively disposition, and not so much addicted to drunkenness as sailors generally are. It was stated that in the early part of his life, he was in the capacity of a groom, when, it appears, he had been thrown from a horse, and had fallen upon his head with some degree of violence; but that during two years' service in the Christian, he had never made any complaint. The immediate consequences of that acci. dent could not be ascertained.

\section{DISSECTION.}

On the 17th March the body was received at the hospital for interment, when I embraced the opportunity of examining the state and appearances of the brain.

Upon inverting the scalp over the eyes and occiput, a slight degree of extravasation of blood was discovered a little above the superciliary ridge, where the blow had been received, but the bone was uninjured. The upper part of the skull being 
forced off, and the dura mater exposed to view, its vessels were found unusually distended. Between the os occipitis and posterior lobe of the right hemisphere, resting on the dura mater, about half an ounce of coagulated blood was found; which, by its remoteness from the injured part, was owing, perhaps, to the rupture of a small vessel, preternaturally distended with blood, a short period previous to dissolution. On removing the dura mater, the vessels of the pia mater were also unusually turgid.

The right hemisphere, being divided on a level with the corpus callosum, and the ventricle exposed by another incision, six drachms of a colourless fluid escaped; and on examining this cavity, a portion of a small encysted tumor was found projecting into it at its anterior cornu. The tumor was imbedded in the corpus callosum, and a considerable part of it rested on the anterior crus of the fornix. It was of the size of a garden bean, felt smooth externally, and when pressed between the finger and thumb, communicated the sensation of its being filled with a cartilaginous substance.

Its connexions were traced to the falx cerebri, and internal carotid artery, by the side of the sella Turcica; and some very minute filaments of vessels were also traced in the direction of the ophthalmic artery, where it enters the foramen. There was neither the appearance of pus nor inflammation in 
the vicinity of the tumor, and the surrounding brain exhibited no mark whatever of morbid affection.

The left ventricle contained nearly two drachms of a colourless fluid, similar to that found in the right; but every other part of the brain and cere. bellum appeared to be wholly free from disease.

When the tumor was removed, and suspended by its numerous vessels, all of which entered together at one point, it exactly resembled a garden bean that had been planted a sufficient time for the radical to have attained length to suspend it by, the vessels entering where the radicle shoots.

The cyst was opake and very strong, and contained a smaller cyst, the size of a barleycorn, imbedded in a semi-medullary and adipose substance, studded with minute portions of bony matter. The small cyst was filled with a piece of bone of an irregular round shape, composed of layers, which the preparation clearly demonstrates from a portion of the outer layer having been broken off by the instrument in laying open the cyst.

That the tumor had existed a considerable period antecedent to the accident which was the immediate cause of the man's death, there is little doubt; and that the preternatural appearances ob- 
served on dissection, independently of the tumor, were sufficient to account for his dissolution, appears to me equally certain.

This case, therefore, seems to evince, in addition to the evidence already existing upon this subject, that a morbid structure of the brain may exist for a long time, without much inconvenience to the patient, or the slightest interruption to the various animal functions; and, with other cases on record*, is, in my estimation, sufficient to authorize the conclusion, that a variety of chronic, and other affections of the head, are referable to blows or falls received in early life; when, probably, the accident that originally occasioned them had wholly escaped recollection. If I mistake not, a similar observation was made by a gentleman present at the reading of my former paper on this subject to the Society.

How long Dawson might have lived with the tumor on his brain, had the accident of which he died not occurred, is matter of mere speculation.

- See Dr. Lettsom's case in the Medical Memoirs before quoted; Sir Gilbert Blane's, in the $2 \mathrm{~d}$ vol. of the Transactions of a Society for the Improvement of Medical and Chirurgical Knowledge ; and Serjeant Turnfield's case, related in this volume, page 191 . 